\title{
A Propósito do Artigo "Indicações para Cesarianas num Hospital Terciário Durante 7 Anos"
}

\author{
Regarding the Article "Indications for Cesarean Deliveries \\ During a 7-Year Period in a Tertiary Hospital"
}

Cristina GUERREIRO'1

Acta Med Port 2013 Nov-Dec;26(6):630-632

Palavras-chave: Cesarianas; Gravidez; Complicações da Gravidez; Hospital Terciário; Portugal.

Keywords: Cesarean Section; Pregnancy; Pregnancy Complications; Portugal; Tertiary Care Centers.

A cesariana é a intervenção cirúrgica mais praticada no Mundo, estimando-se que, anualmente, são realizadas cerca de 20 milhões de cesarianas. ${ }^{1,2}$ Nos países desenvolvidos, tem-se assistido nos últimos 30 anos a um aumento substancial e insustentável da taxa de cesariana, sendo de sublinhar que a melhoria da taxa de mortalidade materna e da morbilidade e mortalidade perinatais não se correlaciona com o tipo de parto.

Os Estados Unidos têm uma taxa de cesariana muito elevada, acima dos $30 \%$, a que não é alheia a pressão exercida sobre os médicos, em termos legais. Na Europa, a taxa de cesariana média é de cerca de $20 \%$, com assimetrias marcadas, verificando-se que são os países do Sul os que têm as taxas mais elevadas. A título de exemplo, em 2007, a taxa mais baixa registou-se na Holanda, com $14 \%$, e a mais elevada foi a de Itália, com $40 \%{ }^{3}$

Portugal tem uma das taxas mais elevadas, entre os membros da União Europeia, apenas superada pela Itália, pela Turquia e pela Grécia. Além de elevada, tem sido progressivamente crescente. No Serviço Nacional de Saúde, aumentou 6 pontos percentuais entre 2001 e 2007, com novo aumento até 2009 , ano em que a taxa de cesariana se situou em $36,4 \%$. Estima-se que a taxa no sector privado seja superior - cerca do dobro.

Com o objectivo de reflectir e implementar medidas conducentes a resultados mais equilibrados, foi criada a Comissão Nacional para a Redução da Taxa de Cesariana (CNRTC),a qual pretende criar um consenso nacional sobre a classificação dos motivos das cesarianas e implementar a estandardização dos dados clínicos obstétricos.

\section{Os riscos da cesariana}

Existe uma opinião generalizada, partilhada por amplos sectores da população, de que a cesariana é a melhor forma de ter 'um parto seguro'. É importante que esta percepção - errada - acerca do risco, seja objecto de uma informação mais esclarecida e fundamentada.

Existem indicações para a realização de uma cesariana e a banalização deste tipo de parto acarreta consequências negativas para a saúde reprodutiva da Mulher. Para além dos riscos inerentes ao procedimento cirúrgico, condiciona o futuro reprodutivo da mulher, aumentando o risco de placenta previa, abruptio placentae, acretismo placentar e rotura uterina em gestações futuras. Em grávidas com 4 cesarianas, o risco de placenta acreta é superior a $60 \%$.

Para o recém-nascido, uma cesariana electiva sem uma indicação clínica clara, também não é a melhor opção. $\mathrm{O}$ trabalho de parto aumenta a actividade do sistema imunitário do feto, estando publicados vários estudos acerca das consequências, a médio e longo prazo, da cesariana electiva. Sugerem, esses estudos, que as crianças nascidas de cesariana, sem trabalho de parto, têm um risco aumentado de diabetes mellitus tipo 1 , asma, alergias e distúrbios gastro-intestinais. ${ }^{4}$

\section{Como reduzir a taxa de cesariana}

Uma estratégia sólida para a redução do número de cesarianas deve apoiar-se na prestação de uma adequada informação às grávidas e balizar-se na definição clínica criteriosa e individualizada do risco para o parto. Tem, como pressupostos, a sustentabilidade de equipamento humano treinado, e a universalidade da analgesia do parto nos Serviços de Obstetrícia e Maternidades.

O treino no parto instrumentado é um factor que contribui para a redução do número de cesarianas e deve ser uma preocupação constante nos Serviços de Obstetrícia que formam novos especialistas. Um dos factores contributivos para a elevada taxa de cesariana nos Estados Unidos foi o rápido declínio do parto vaginal instrumentado ${ }^{5}$ catalizado em 1998 pela preocupação com os riscos potenciais da ventosa. ${ }^{6}$ Estudos retrospectivos, robustos e de boa qualidade, não demonstraram esse risco, evidenciando incidências idênticas de hemorragia intracraniana para a ventosa e para a cesariana ${ }^{7}$ e menor morbilidade neurológica associada ao fórceps, comparativamente com a ventosa e a cesariana. ${ }^{8} \mathrm{Um}$ estudo recentemente publicado, ${ }^{9}$ avaliou a relação entre via de parto e desfechos neonatais adversos, num período de 10 anos, em gravidez de termo, única, com feto em apresentação cefálica. A morbilidade foi idêntica para o parto vaginal instrumentado e para a cesa-

1. Assistente Graduada de Ginecologia e Obstetrícia. Maternidade Dr. Alfredo da Costa (Centro Hospitalar Lisboa Central). Lisboa. Portugal. Recebido: 04 de Dezembro de 2013 - Aceite: 05 de Dezembro de 2013 | Copyright $\odot$ Ordem dos Médicos 2013 
riana em trabalho de parto.

Dos 10 grupos da classificação de Robson, ${ }^{10}$ o que mais contribui para um aumento do número de cesarianas é o grupo da cesariana anterior. Evitar a primeira cesariana, em nulíparas de baixo risco, é uma estratégia crucial para reduzir a repetição de cesariana em gestações subsequentes. ${ }^{11}$

Outra medida para obviar à realização de cesarianas evitáveis, é ser criterioso nas induções do trabalho de parto, restringindo as mesmas às indicações médicas, particularmente em situações com índice de Bishop desfavorável. A indução do trabalho de parto por conveniência materna ou do médico, sem uma indicação clínica, só deverá ser realizada depois das 39 semanas e com índice de Bishop superior a 8 , particularmente em nulíparas. Deve ser revisto o conceito de 'indução falhada' e os critérios de trabalho de parto estacionário. A medida mais eficaz para reduzir a morbilidade associada à cesariana, é evitar a primeira cesariana. $^{12}$

Liberalizar o parto vaginal em mulheres com uma cesariana anterior, é igualmente importante para reduzir o número de cesarianas e a morbilidade associada. Curiosamente, os hospitais com taxas mais elevadas de parto vaginal em mulheres com cesariana anterior, são os que têm também taxas mais baixas de cesariana primária. ${ }^{13} \mathrm{Al}-$ gumas das razões para esta associação, serão uma cultura organizacional que privilegia o parto vaginal, a existência de profissionais treinados e de condições logísticas favoráveis à resolução, com eficácia, de situações de emergência obstétrica.

Em 2001, Robson sublinhou que a questão fulcral não é saber se a taxa de cesariana é demasiado alta ou demasiado baixa, mas sim se o parto por cesariana tem (ou não tem) uma indicação adequada, mediante uma correcta avaliação clínica. É expectável que a taxa de cesariana seja mais elevada em centros terciários, de apoio perinatal diferenciado, os quais centralizam situações clínicas de maior complexidade e vigiam gestações de alto risco.

Os protocolos de cada Serviço de Obstetrícia têm uma influência decisiva na taxa de cesariana. É neste contexto que se insere o artigo publicado na Acta Médica Portuguesa: 'Indicações para cesarianas num hospital terciário durante 7 anos'. Trata-se de uma avaliação retrospectiva sobre as indicações das cesarianas no Departamento de Obstetrícia do Hospital de Santa Maria, durante um período em que se implementaram modificações na prática clínica, que evidenciam uma reflexão e uma preocupação pelo rigor no desempenho da Obstetrícia.

Os autores apresentam um trabalho cujo tema é actualmente da maior relevância, com resultados que são um desafio positivo para outros Serviços de Obstetrícia. No período analisado, houve uma descida de $3,3 \%$ na taxa de cesariana, devido ao decréscimo na primeira cesariana, resultante de modificações na prática clínica do Departamento: versão cefálica externa e indução do trabalho de parto só a partir das 41 semanas, em gravidez de baixo risco. E, ainda, a realização de prova de trabalho nalguns casos de patologia materno-fetal e em grávidas com cesariana anterior.

A diferenciação em medicina materno-fetal e a experiência em obstetrícia deste centro terciário são, certamente, factores que contribuíram para os resultados, deixando um convite à reflexão sobre a importância do treino médico e da diferenciação em Obstetrícia. É também de sublinhar a taxa de sucesso de $44,4 \%$ na versão cefálica externa, resultando numa diminuição do número de cesarianas por apresentação pélvica.

Relativamente às cesarianas em trabalho de parto, os novos critérios de trabalho de parto estacionário poderão evitar algumas cesarianas realizadas por essa indicação. Atingir uma maior acuidade diagnóstica para o sofrimento fetal, continua a ser um enorme desafio em Obstetrícia, pela complexidade dos sinais clínicos e pelas consequências que podem advir de uma intervenção protelada.

\section{Cesariana 'a pedido'}

A cesariana 'a pedido' é uma cesariana primária, realizada antes do início do trabalho de parto, por opção da mulher grávida, na ausência de indicações maternas ou fetais. A sua incidência é mal conhecida, bem como o seu contributo para a taxa de cesariana. Nos Estados Unidos, estima-se que totalize $2,5 \%$ de todos os partos.

É muito frequente que o pedido de uma cesariana se baseie em informação errada acerca dos riscos e benefícios do procedimento. É fundamental que o médico disponibilize tempo e espaço para prestar uma informação correcta e objectiva, que dissipe alguns dos mitos mais frequentes,,$^{14}$ nomeadamente a dor associada ao trabalho de parto, as consequências para o pavimento pélvico e a segurança para o feto. Nas situações em que, apesar de uma correcta informação, abrangendo os riscos e as consequências para o futuro reprodutivo, a decisão da Mulher se mantém, a realização da cesariana não deve ser programada antes das 39 semanas. ${ }^{15}$ O Colégio Americano de Obstetrícia não recomenda a cesariana 'a pedido' para mulheres que desejem ter muitos filhos, devido ao risco exponencial de placenta previa e placenta acreta em gestações futuras.

As conclusões de diversos inquéritos realizados para aferir as preferências das mulheres acerca da via de parto, têm evidenciado uma preferência maioritária pelo parto vaginal. ${ }^{16-18}$

Em Itália, o país europeu com a taxa mais elevada de cesariana, a cesariana 'a pedido' perfez apenas $6,4 \%$ das cesarianas realizadas num hospital universitário, no período compreendido entre 1996 e 2000 . Um inquérito realizado em Itália concluiu que 4 em cada 5 mulheres preferiam ter um parto vaginal, se pudessem optar. Nas que preferiam a cesariana, as razões subjacentes a essa escolha eram o medo da dor, a conveniência em poder planear o dia do parto e a percepção de que seria menos traumático para o feto. Questionadas acerca de quem mais poderia influenciar ou alterar a sua escolha, o obstetra figurava em primeiro lugar, seguindo-se os familiares e os amigos. ${ }^{19}$ 


\section{Em conclusão:}

A cesariana é um procedimento cirúrgico com riscos e consequências negativas para o futuro reprodutivo da Mulher. A elevada taxa de cesariana, actualmente verificada, pode ser corrigida sem prejuízo dos resultados perina-

\section{REFERÊNCIAS}

1. Betrán AP, Merialdi M, Lauer JA, Bing-Shun W, Thomas J, Van Look P et al: Rates of cesarean section: analysis of global, regional and national estimates. Paediatr Perinat Epidemiol. 2007; 21:98-113.

2. Gibbons L, Belizan JM, Lauer JA. Inequities in the use of cesarean section deliveries in the world. Am J Obstet Gynecol. 2012;206:331.e1-19.

3. Organization for Economic Co-Operation and Development (OECD) Health at a glance 2009. OECD indicators. Paris: OECD; 2009.

4. Romero R, Korzeniewski SJ. Are infants born by elective cesarean delivery without labor at risk of developing immune disorders later in life? Am J Obstet Gynecol. 2013;208:243-6.

5. Oliphant SS, Jones KA, Wang L, Bunker CH, Lowder JL. Trends over time with commonly performed obstetric and gynecologic inpatient procedures. Obstet Gynecol. 2010;116:926-31.

6. US Food and Drug Administration. Public health Advisory: need for caution when using vacuum assisted delivery devices. Rockville: FDA Center for Devices and Radiological Health; 1998.

7. Towner D, Castro MA, Eby-Wilkens E, Gilbert WM. Effect of mode of delivery in nulliparous women on neonatal intracranial injury. $\mathrm{N}$ Engl $\mathrm{J}$ Med. 1999;341:1709-14.

8. Werner EF, Janevic TM, Illuzzi J, Funai EF, Savitz DA, Lipkind HS. Mode of delivery in nulliparous women and neonatal intracranial injury. Obstet Gynecol. 2011;118:1239-46.

9. Walsh A, Robson M, McAuliffe F. Mode of delivery at term and adverse neonatal outcomes. Obstet Gynecol. 2013;121:122-28.

10. Robson MS. Classification of cesarean sections. Fetal Maternal Rev. 2001;12:23-39.

11. Delbaere I, Cammu H, Martens E, Tency I, Martens G, Temmerman tais. O rigor na avaliação e na decisão clínica, a informação e educação para a saúde, a analgesia do parto e o treino obstétrico, são factores decisivos para diminuir o número de cesarianas e a morbilidade associada.

M. Limiting the cesarean section rate in low risk pregnancies is key to lowering the trend of increased abdominal deliveries: an observational study. BMC Pregnancy Childbirth. 2012;12:3.

12. Spong CY, Berghella V, Wenstrom KD, Mercer BM, Saade GR. Preventing the first cesarean delivery: summary of a joint Eunice Kennedy Shriver National Institute of Child Health and Human Development, Society for Maternal-Fetal Medicine, and American College of Obstetricians and Gynecologists Workshop. Obstet Gynecol. 2012;120:1181-93.

13. Rosenstein MG, Kuppermann M, Gregorich SE, Cottrell EK, Caughey $A B$, Cheng YW. Association between vaginal birth after cesarean delivery and primary cesarean delivery rates. Obstet Gynecol. 2013;122:1010-17.

14. ACOG Committee Opinion No. 578: Elective surgery and patient choice. Obstet Gynecol. 2013;122:1134-8.

15. American College of Obstetricians and Gynecologists. ACOG committee opinion no. 559: Cesarean delivery on maternal request. Obstet Gynecol. 2013;121:904-7.

16. Mould TA, Chong S, Spencer JA. Women's involvement with the decision preceding their cesarean section and their degree of satisfaction. $\mathrm{Br}$ J Obstet Gynecol. 1996;103:1704-77.

17. Usha Kiran TS, Jayawickrama NS. Who is responsible for the rising of cesarean section rate? J Obstet Gynaecol. 2002;22:363-5

18. Wilkinson $\mathrm{C}$, Mcllwaine $\mathrm{G}$, Boulton-Jones $\mathrm{C}$, Cole $\mathrm{S}$. Is a rising ceasarean section rate inevitable? Br J Obstet Gynaecol. 1998;105:45-52.

19. Torloni MR, Betrán AP, Montilla P, Scolaro E, Seuc A, Mazzoni A, et al. Do Italian women prefer cesarean section? Results from a survey on mode of delivery preferences. BMC Pregnancy Childbirth. 2013;13:78. 


\section{A Propósito do Artigo "Indicações para Cesarianas num Hospital Terciário Durante 7 Anos" \\ Acta Med Port 2013:26:630-632}

Publicado pela Acta Médica Portuguesa, a Revista Científica da Ordem dos Médicos

Av. Almirante Gago Coutinho, 151

1749-084 Lisboa, Portugal.

Tel: +351218428215

E-mail: submissao@actamedicaportuguesa.com

www.actamedicaportuguesa.com

ISSN:0870-399X | e-ISSN: 1646-0758

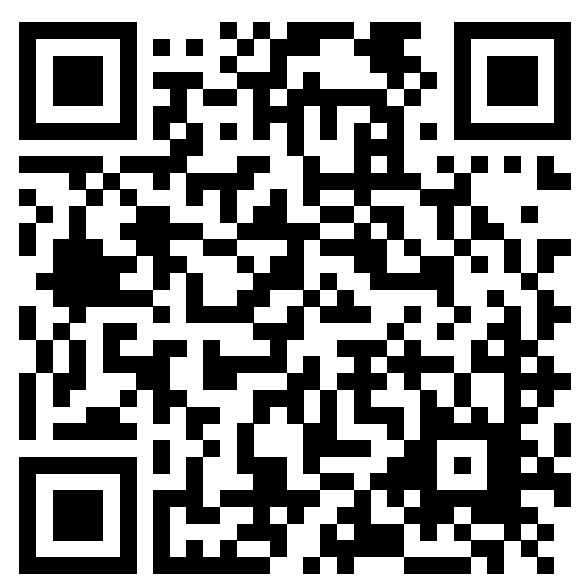

